\title{
Energy Management Strategy for High-Altitude Solar Aircraft Based on Multiple Flight Phases
}

\author{
Mou Sun $\mathbb{D}^{1},{ }^{1}$ Chuan Shan, ${ }^{1}$ Kang-wen Sun $\mathbb{D D}^{2,3}$ and Yu-hong Jia ${ }^{1}$ \\ ${ }^{1}$ School of Aeronautic Science and Engineering, Beihang University, Beijing 100191, China \\ ${ }^{2}$ Institute of Unmanned System, Beihang University, Beijing 100191, China \\ ${ }^{3}$ Yunnan Innovation Institute, Beihang University, Kunming 650233, China \\ Correspondence should be addressed to Kang-wen Sun; sunkw100@buaa.edu.cn
}

Received 11 October 2020; Revised 10 November 2020; Accepted 27 November 2020; Published 9 December 2020

Academic Editor: Kai Wang

Copyright (C) 2020 Mou Sun et al. This is an open access article distributed under the Creative Commons Attribution License, which permits unrestricted use, distribution, and reproduction in any medium, provided the original work is properly cited.

Making use of solar energy to fly is an up-and-coming technology in the human aviation field since solar energy is renewable and inexhaustible, and more and more attention and efforts have been directed to the development of high-altitude solar aircraft (HSA). Due to the technical constraints of the rechargeable battery, the HSA must carry sufficient batteries to meet the flight power consumption at night, which seriously limits the flight endurance of HSA. To solve this contradiction, the paper has proposed a new energy management strategy (EMS) of multiple flight phases for HSA based on the gravitational energy storage and mission altitude, which aims to achieve the goal of long-endurance flight for HSA. The integrated model of this new EMS includes the aerodynamic model, the kinematic model, the solar irradiation model, the battery model, and the energy management model. Compared with the current EMS of level flight, the flight path of HSA in the new EMS has been divided into five phases: the lower altitude level flight at night, the maximum power ascending for mission altitude, the level flight at mission altitude, the maximum power ascending for higher altitude, and the longest gliding endurance. At last, the calculation of the new EMS for Zephyr 7 is studied by MATLAB/Simulink, and the calculation results indicate that about $22.9 \%$ of energy surplus can be stored in battery with the new EMS for Zephyr 7 compared with the current EMS, which is equal to reducing the rechargeable battery weight from $16.0 \mathrm{~kg}$ to $12.3 \mathrm{~kg}$. Besides, the results of simulation in the four seasons also show that the new EMS is a very promising way to achieve the long-endurance goal for high-altitude HSA when the flight conditions satisfy some constraints like the deficiency of solar flux and the limit of battery mass.

\section{Introduction}

Nowadays, environmental pollution and energy crisis are the tough challenges that the world and humanity are facing [1]. Due to the rapid development of solar cell technology, solar energy can gradually play a leader role in exploring the field of renewable and clean energy [1-3]. Therefore, making use of solar energy to fly is a research hotspot, which has attracted a lot of research groups all around the world during the past few years [4-10].

Since the solar energy is renewable and inexhaustible, it has the potential to redefine and increase the flight endurance of aircraft. In the last ten years, many countries and research teams have made great efforts to develop the high-altitude solar aircraft (HSA) [2, 6-12] for the reason that the HSA have the ability to stay over a wide area at high altitude for long endurance; they can function as geostationary satellites, but they are much economical $[13,14]$. They are ideally suited to be widely employed in many applications such as natural monitoring, border patrol, planetary exploration, communications relay, remote sensing, field investigations, network services, and electronic warfare [14]. Over the past years, there are two series of highaltitude long-endurance (HALE) solar-powered aircraft programs which aim to achieve the goal of the HALE flight: Zephyr and ERAST $[7,15,16]$. However, due to the technical limitations of rechargeable battery and structure, none of these aircraft has achieved the HALE flight in real sense. As a 
result of those pioneering and advanced research studies and experiments, scholars and researchers have gradually realized that the key factor restricting the long-endurance flight of HSA is the problem of how to meet the power consumption at night with the constraints on the weight of the battery $[2,14,16]$. Under the current technique level, the weight of rechargeable battery occupies about $45 \%$ of the total mass of aircraft $[3,11]$. Meanwhile, the HSA technologies like solar cell conversion efficiency and battery specific capacity are unable to improve rapidly. Therefore, the regenerative power technology such as energy management strategy (EMS) is the key to achieving long-endurance flight for HSA.

In recent decades, some studies have focused on the regenerative power technologies about drones and their applications. The studies $[17,18]$ show that the efficiency and the attitude angles of the solar cell directly affect the energy production power for the applications of solar drone platform. Especially, Scheiman et al. have built an Unmanned Aerial Vehicle (UAV) with a variety of solar cell technologies in the wing. In order to extend flight endurance, the solar cells mainly include thin flexible GaAs, high efficiency $\mathrm{Si}$, and triple junction $\mathrm{InGaP} / \mathrm{GaAs} / \mathrm{Ge}$. As a result, the UAV power management system is experimentally verified to perform well. The studies $[19,20]$ conducted thorough analytical research on rechargeable battery based on energy system of drone. These works are significant in the selection of battery and the estimation of the battery capacity for HSA. The studies [21-26] researched energy system and EMS for HSA and airships, and some important conclusions have been obtained. Some of these designed energy systems which had been experimentally tested and verified have made significant and pioneering contributions to the development for solar drone EMS. In addition, many scholars and researchers have studied and improved the EMS in other fields to increase the power efficiency of energy system, which include the hybrid vehicle, the air conditioning, and the light electric bus [27-31]. However, none of these research papers have studied the EMS for HSA in real sense based on multiple fight phases. As is known, the HSA need to have a multiple-phase flight process in engineering applications, which generally includes executing mission in higher altitude, cruising in lower altitude, and flight paths for gravity energy storage. In the higher altitude level flight during the daytime, the HSA can perform mission like regional residency and circle hover, as well as charging the battery smoothly with the electricity generated by the solar cells. In the lower altitude at night, the HSA can achieve the goal of long endurance with lower power consumption. Meanwhile, those two different level flight altitudes are also beneficial to realize the gravity energy storage strategy. Therefore, the above-mentioned multiple flight stages should be designed in the EMS for long-endurance HSA, and to the best of our knowledge, almost none of these energy systems is introduced and involved in the published literature.

This article is inspired by these considerations and a study [32] that proposes the EMS based on the idea that the surplus solar energy can be stored in gravitational potential during the daytime. The contributions of this study can be summarized as follows:

(1) The new EMS model based on multiple flight phases for HSA is proposed, which is more suitable for the day and night cycle flight in engineering applications.

(2) The design of mission altitude for level flight in the multiple flight phases indicates and provides the possibility that HSA perform missions such as the regional residence, the straight back, and the circle hover, and the HSA can function as geostationary satellites.

(3) In order to calculate the new EMS for HSA, an integrated MATLAB/Simulink calculation program including the aerodynamic model, the kinematic model, the solar flux model, the battery model, and the EMS model is established.

(4) Due to the particularity of energy system, the research results also show that a suitable EMS can be more beneficial to long endurance for HSA on condition of lower solar irradiation and shorter daytime.

\section{Models and Methods}

A typical HSA energy system [21, 22] mainly includes solar cell, voltage converter with maximum power point tracking (MPPT) function, energy management system, and rechargeable battery with management system, as shown in Figure 1. In the daytime, the electricity generated by solar cells on the wings can directly supply the power through the energy management system. Meanwhile, the excess solar energy will charge the battery. At night, the HSA power is all provided by the rechargeable battery. As a result, the HSA could fly uninterruptedly at day and night for long-endurance goal.

Based on this energy system of HSA, the models and methods used in the paper will be introduced and established as follows.

2.1. Aircraft Kinematic and Aerodynamic Models. As shown in Figure 2, the study defines the $O_{g} X_{g} Y_{g} Z_{g}$ as the earthfixed frame, and the aircraft body-fixed frame is $O_{b} X_{b} Y_{b} Z_{b}$, where $O_{g} X_{g}, O_{g} Y_{g}$, and $O_{g} Z_{g}$ represent the north, the west, and the straight directions through the ground. $O_{b} X_{b}$ is located in a symmetric position parallel to the fuselage axis and points forward, $O_{b} Y_{b}$ points perpendicularly to the right of the plane of symmetry, and $O_{b} Z_{b}$ obeys the right hand axis rule and is perpendicular to $\mathrm{O}_{b} X_{b}, O_{b} Y_{b}$.

In this paper, the study of the new EMS mainly focuses on the energy supply and consumption characteristics of the energy system for HSA. Thus, the three-dimensional dynamic equations of HSA can be simplified as follows [33]: 


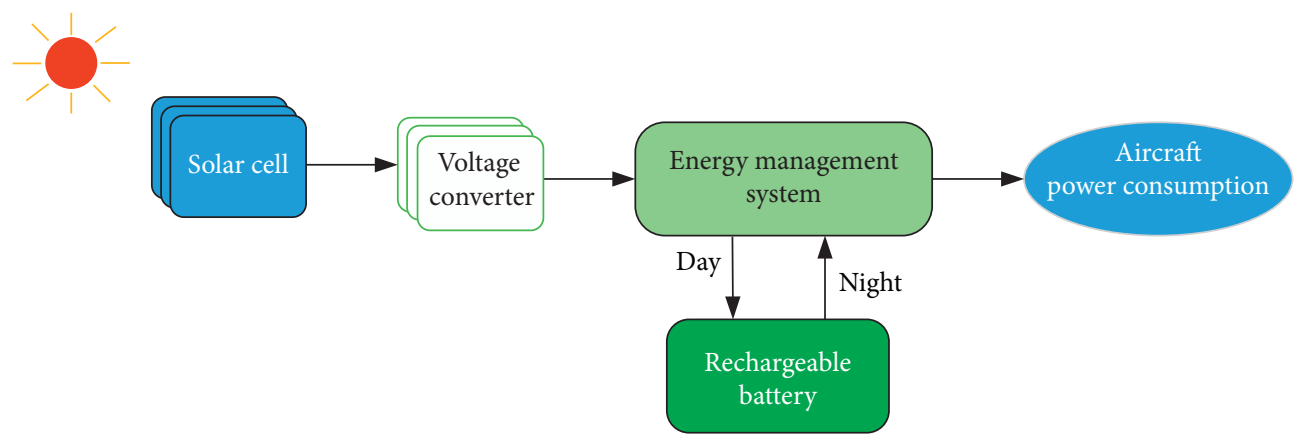

FIGURE 1: Schematic picture of energy system for high-altitude solar aircraft.

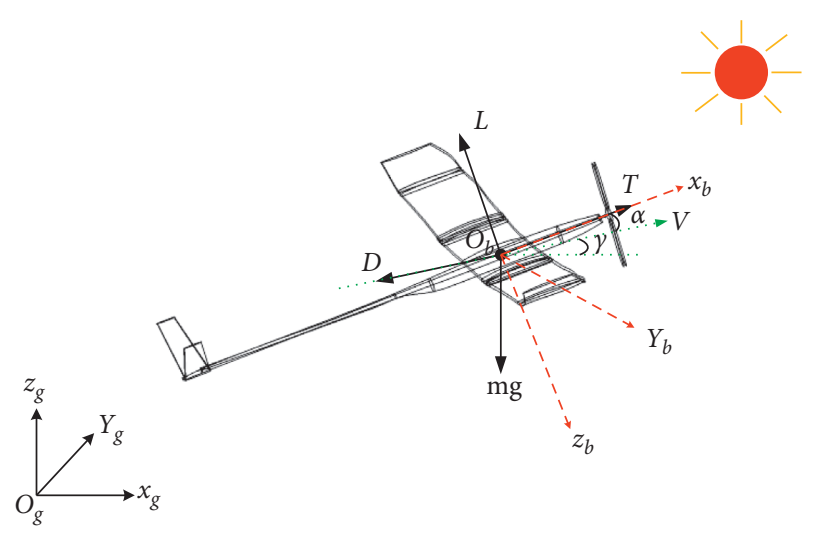

FIGURE 2: Schematic diagram of three-dimensional forces acting on solar-powered aircraft.

$$
\left\{\begin{array}{l}
\frac{\mathrm{d} v}{\mathrm{~d} t}=\frac{T \cos \alpha}{m}-\frac{D}{m}-g \cdot \sin \gamma, \\
\frac{\mathrm{d} \gamma}{\mathrm{d} t}=\frac{T \sin \alpha}{m v}+\frac{L}{m v} \cos \psi-\frac{g}{v} \cos \gamma \\
\frac{\mathrm{d} \varphi}{\mathrm{d} t}=\frac{L}{m v \cos \gamma} \sin \psi, \\
\frac{\mathrm{d} x}{\mathrm{~d} t}=v \cos \gamma \cos \varphi, \\
\frac{\mathrm{d} y}{\mathrm{~d} t}=v \cos \gamma \sin \varphi, \\
\frac{\mathrm{d} h}{\mathrm{~d} t}=v \sin \gamma
\end{array}\right.
$$

where $x, y$, and $h$ are the HSA north, east, and altitude positions in the earth-fixed frame, $v$ is the HSA speed, $\psi$ is the roll angle of the HSA, $\varphi$ is the yaw angle, $\gamma$ is the flight path angle, $m$ is the total mass of HSA, and $g$ is the gravity acceleration. $T, L$, and $D$ mean the thrust force, aerodynamic lift, and drag forces of the HSA.
As shown in Figure 2, the angle of attack $\alpha$ is the angle between velocity vector direction and $O_{b} X_{b}$ axis direction, and the pitching angle $\theta$ is the sum of $\alpha$ and $\gamma$.

Generally speaking, the aerodynamic forces $L$ and $D$ of the HSA can be expressed in the following formula:

$$
\left\{\begin{array}{l}
L=\frac{1}{2} \rho v^{2} \cdot S_{W} C_{L}, \\
D=\frac{1}{2} \rho v^{2} \cdot S_{W} C_{D},
\end{array}\right.
$$

where $S_{W}$ is the wing area, $C_{D}$ and $C_{L}$ are the drag and lift coefficients, and $\rho$ is the air density.

2.2. Solar Irradiation Model. As is known, the solar radiation intensity is related to many factors, including the date, latitude, and longitude. The paper summarizes a lot of literature $[2,3,22,32-34]$ about the calculation of solar flux, and the solar power per square meter in the earth-fixed frame can be calculated by the following formula:

$$
\left\{\begin{aligned}
P_{S} & =I_{\mathrm{SC}} \cdot \tau \cdot \sin \left(H_{S}\right), \\
I_{\mathrm{SC}} & =I_{0} \cdot\left(\frac{1+\varepsilon \cdot \cos (2 \pi(n-4) / 365)}{1-\varepsilon^{2}}\right)^{2}, \\
\sin \left(H_{S}\right) & =\sin \phi \sin \delta+\cos \phi \cos \delta \cos (\pi-\pi \cdot t / 12), \\
\delta & =\frac{23.45 \pi}{180} \sin \left(360 \frac{284+n}{365}\right),
\end{aligned}\right.
$$

where $P_{S}$ is the solar power in per square meter, $I_{0}$ is the solar irradiance constant that has a value of $1367 \mathrm{~W} / \mathrm{m}^{2}, \tau$ is the transmittance factors, $H_{S}$ is the solar elevation angle as shown in Figure 3, $\varepsilon$ is the eccentricity ratio of earth, $n$ is the day number of the year, $\phi$ is the location latitude, $t$ is the true local solar time, and $\delta$ is the solar declination angle.

Equation (3) shows a more concise and complete calculation about the $P_{S}$ in the earth-fixed frame. In particular, the transmittance factor $\tau$ is affected by atmospheric 


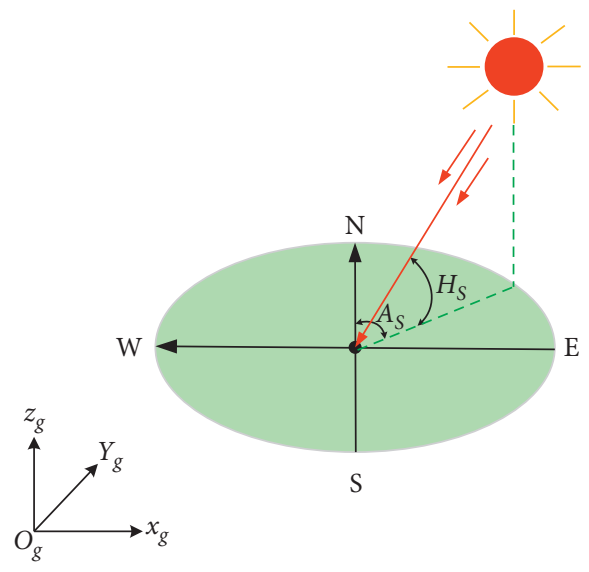

FIgURE 3: Schematic diagram of the sun azimuth and the elevation angle.

transparency and so on. That includes the modifying factors, the atmospheric pressure of different altitudes, and the air mass ratio which are described in literature [21]. The parameter $t$ is related to the standard time and the longitude of the location. Those parameters in integrated equation (3) can be introduced and calculated by the formulas in the literature above, which are not be described in detail here.
With the HSA transforming the flight altitude and attitude during the daytime, the solar energy on the wings surface generated by solar cells will always change in time. Therefore, a relatively accurate solar flux model about the attitude and altitude of the HSA needs to be set up to calculate solar energy collection. Based on the display in Figure 2 and the calculation of $P_{S}$ in the earth-fixed frame from (3), the solar flux model for HSA can be obtained by the coordinate conversion principle between the bodyfixed frame $O_{b} X_{b} Y_{b} Z_{b}$ and the earth-fixed frame $\mathrm{O}_{g} X_{g} Y_{g} Z_{g}$.

Firstly, the $P_{S}$ in each direction calculation $I_{X g}, I_{Y g}$, and $I_{Z g}$ (positive and negative represent directions) can be expressed by the following formula:

$$
\left\{\begin{array}{l}
I_{X g}=P_{S} \cdot \cos H_{S} \cdot \cos A_{S} \\
I_{Y g}=P_{S} \cdot \cos H_{S} \cdot \sin A_{S} \\
I_{Z g}=P_{S} \cdot \sin H_{S}
\end{array}\right.
$$

where $A_{S}$ is the sun azimuth angle, as shown in Figure 3.

Secondly, based on the coordinate conversion principle, the solar radiation intensities $I_{X b}, I_{Y b}$, and $I_{Z b}$ received in the body-fixed frame can be calculated by the following formula:

$$
\left[\begin{array}{c}
I_{X b} \\
I_{Y b} \\
I_{Z b}
\end{array}\right]=\left[\begin{array}{ccc}
\cos \theta \cos \varphi & \cos \theta \sin \varphi & -\sin \theta \\
\sin \psi \sin \theta \cos \varphi-\cos \psi \sin \varphi & \sin \psi \sin \theta \sin \varphi+\cos \psi \cos \varphi & \cos \theta \sin \psi \\
\cos \varphi \sin \theta \cos \psi+\sin \varphi \sin \psi & \cos \psi \sin \theta \sin \varphi-\sin \psi \cos \varphi & \cos \theta \cos \psi
\end{array}\right]\left[\begin{array}{c}
I_{X g} \\
I_{Y g} \\
-I_{Z g}
\end{array}\right] .
$$

To sum up, the solar energy collection model for HSA can be established into a MATLAB function file for further model integration. This solar flux intensity calculation model of HSA on the wings is related to the longitude, latitude, altitude, date, moment, pitch angle, yaw angle, and roll angle.

2.3. Energy Management Strategy. As shown in Figure 1, the function of energy management system is to control the energy flow for HSA, which mainly includes the battery management system and the switching system of power supply mode. During the daytime, the maximum electrical energy generated by solar cells and voltage converter is divided into two parts: one part directly supplies electricity to the propulsion motors and on-board equipment, and the other part will recharge the battery with excess energy. At night, the power supply mode of the energy management system is that the battery powers the propulsion system and the equipments on board. The efficiency values of each component of the energy system are listed in Table 1 .

Generally speaking, the MPPT function will be embedded in the solar voltage converter. Therefore, the $\eta_{\mathrm{mppt}}$ is the efficiency of converting solar energy into electricity in this study. Besides, it will not distinguish between battery charging and discharging efficiency in general research field. In order to simplify the calculation, this paper assumes that the battery charge and discharge efficiency are equal to 0.98 and the propulsion system efficiency is reasonably given as 0.7 although the efficiencies of motors and airscrew will vary with altitude and Reynolds number. Thus, the thrust $T$ of the propulsion system can be obtained by the following formula:

$$
T=\eta_{\mathrm{ma}} \cdot \frac{P_{\text {prop }}}{v}
$$

where $P_{\text {prop }}$ is the propulsion system power.

\subsubsection{Current Energy Management Strategy Based on Level} Flight. The purpose of EMS for HSA is to realize longendurance flight with the limit of battery mass at night. For most of the reported HSA energy systems, the EMS can be expressed as flying with minimum power consumption with the constraints of lift and weight balance, thrust and drag balance, and energy supply and demand balance, as shown in Figure 4.

Generally, the surplus energy will charge the battery by the current EMS if the output solar power $P_{\text {mpt }}$ of the voltage converter is larger than the power $P_{\text {prop }}$ (as on-board equipment power can be ignored for the $\left.P_{\text {prop }}\right)$. Meanwhile, the insufficient required power will be supplemented by the 
TABLE 1: The efficiency of each component in the energy management system.

\begin{tabular}{lccc}
\hline Components & Symbol & Value & \\
\hline Solar cell & $\eta_{\mathrm{sc}}$ & 0.2 & Units \\
Voltage converter & $\eta_{\mathrm{mppt}}$ & 0.95 & - \\
Battery & $\eta_{b}$ & 0.98 & - \\
Battery manager & $\eta_{\mathrm{bm}}$ & 0.99 & - \\
Motors and airscrew & $\eta_{\mathrm{ma}}$ & 0.7 & - \\
\hline
\end{tabular}
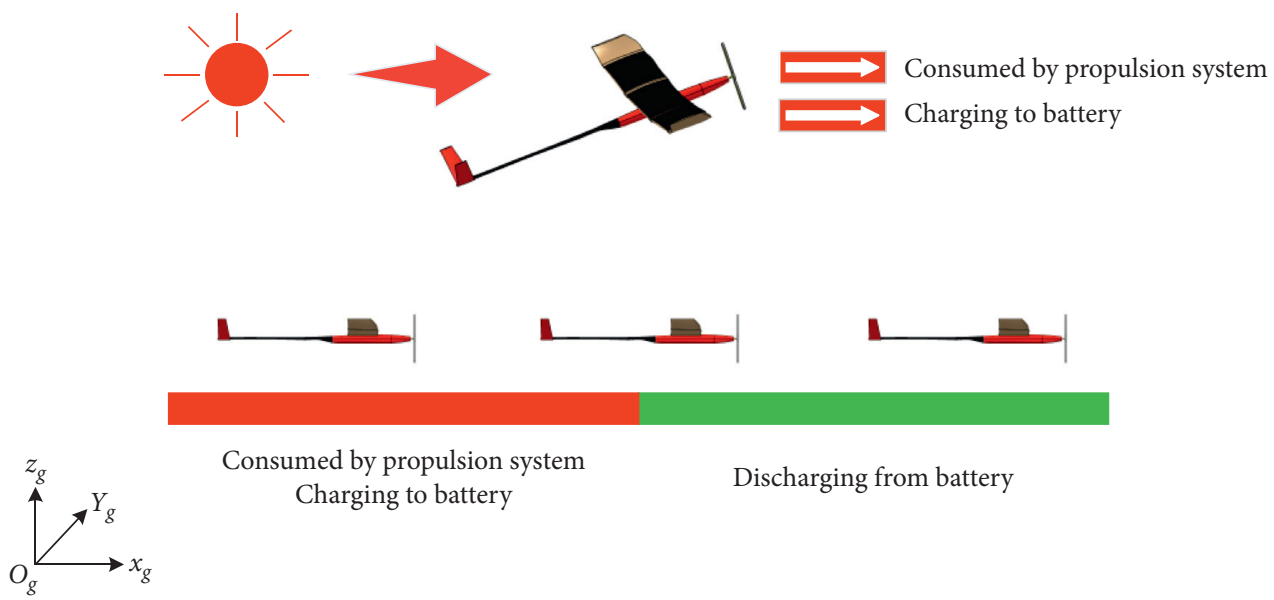

FIgURE 4: Schematic diagram of the current energy management strategy based on level flight.

battery when the $P_{\text {mppt }}$ is less than the $P_{\text {prop. The output }}$ power $P_{\text {mppt }}$ of the voltage converter is calculated as follows:

$$
P_{\mathrm{mppt}}=\left[\begin{array}{c}
I_{X b} \\
I_{Y b} \\
I_{Z b}
\end{array}\right] \cdot S_{\mathrm{SC}} \cdot \eta_{\mathrm{mppt}},
$$

where $S_{\mathrm{SC}}$ is the solar cells area in the wing. For the HSA level flight,

$$
\left\{\begin{array}{l}
\gamma=0, \\
D=T \cdot \cos \alpha \\
L=m g-T \cdot \sin \alpha .
\end{array}\right.
$$

The power consumption of the aircraft in level flight can be obtained as shown in the following formula:

$$
\begin{aligned}
\eta_{\mathrm{ma}} \cdot P_{\text {prop }} & =P_{\text {level }}=T \cdot v=\frac{D \cdot v}{\cos \alpha} \\
& =\frac{C_{D}}{C_{L}^{(3 / 2)}}\left(\frac{m g-T \cdot \sin \alpha}{\cos \alpha}\right) \sqrt{\frac{2(m g-T \cdot \sin \alpha)}{\rho \cdot S_{W}} .}
\end{aligned}
$$

The angle of attack $\alpha$ control command for the HSA flight path can be expressed as

$$
\begin{aligned}
\alpha_{\text {level }}^{*} & =\min _{\alpha_{\min }<\alpha<\alpha_{\max }}\left(P_{\text {prop }}\right) \\
& =\min _{\alpha_{\min }<\alpha<\alpha_{\max }}\left\{\frac{C_{D}}{C_{L}^{(3 / 2)}}\left(\frac{m g-T \cdot \sin \alpha}{\cos \alpha}\right) \sqrt{\frac{2(m g-T \cdot \sin \alpha)}{\rho \cdot S_{W}}}\right\} .
\end{aligned}
$$

Therefore, the current EMS of HSA can be expressed as follows:

$$
\dot{E}=\left\{\begin{array}{lc}
q_{\mathrm{max}}, & \left(P_{\mathrm{mppt}}-P_{\mathrm{prop}}\right) \eta_{b} \eta_{\mathrm{bm}} \geq q_{\mathrm{max}}, \\
\frac{\left.\left(P_{\mathrm{mppt}}-P_{\mathrm{prop}}\right) \eta_{b} \eta_{\mathrm{bm}}, P_{\mathrm{prop}}\right)}{\left(\eta_{b} \eta_{\mathrm{bm}}\right)} & 0 \leq\left(P_{\mathrm{mppt}}-P_{\mathrm{prop}}\right) \eta_{b} \eta_{\mathrm{bm}}<q_{\mathrm{max}}, \\
\frac{-P_{\mathrm{prop}},}{\left(\eta_{b} \eta_{\mathrm{bm}}\right)} & \left(P_{\mathrm{mppt}}-P_{\mathrm{prop}}\right) \eta_{b} \eta_{\mathrm{bm}}<0, \\
0, & P_{\mathrm{mppt}}=0, \\
& Q_{B} \leq Q_{B_{-} \min } \text { or } Q_{B} \geq Q_{B_{-} \max },
\end{array}\right.
$$

where $\dot{E}$ is the battery charge and discharge power (positive means charging; negative means discharging), $q_{\max }$ is the battery maximum charging power, and $Q_{B}$ is the electricity energy in battery.

\subsubsection{Energy Management Strategy Based on Multiple Flight} Phases. As shown in (11), the current EMS of HSA is relatively simple because more attention has been paid to the engineering applications, and the HSA can achieve longendurance flight only by carrying more batteries. However, the more batteries will increase the power consumption during the level flight. Thus, the current EMS which simply relies on battery to store energy is not an effective way of 
using solar energy, and the research of new EMS based on multiple flight phases including the lower cruising altitude and higher mission altitude for HSA will be particularly important.

The flight path of EMS based on multiple flight phases proposed in this paper is mainly divided into five stages, as shown in Figure 5. The design of mission altitude $h_{2}$ is beneficial to the engineering applications, and this is also conducive to charging the battery smoothly. Besides, it can be seen that the HSA can also execute a flight mission in a stable process in the mission altitude $h_{2}$ with the lower wind speed. On the other hand, the HSA can cruise in lower power consumption due to the lower altitude $h_{1}$ at night. At the same time, some missions like surveillance can be implemented at this altitude. Therefore, the above-proposed longendurance flight path is significant for HSA in engineering applications, and the details of the phases are as follows.

(1) Phase 1: Lower Power Level Flight at Night. The stage of flight starts at time $t_{4}$ at the night before when the HSA reaches the altitude $h_{1}$, and finishes at time $t_{0}$ when the output solar power $P_{\text {mppt }}$ of the voltage converter is larger than the required power $P_{\text {prop }}$ in $h_{1}$ altitude. During this stage, the HSA is only supplied by battery for cruising. The required power for level flight of the HSA can be gained by (9). The proposed EMS at this stage can be represented by the following formula:

$$
\dot{E}= \begin{cases}\frac{-P_{\text {prop }}}{\left(\eta_{b} \eta_{\mathrm{bm}}\right)}, & Q_{B}>Q_{B_{-} \min }, \\ 0, & Q_{B} \leq Q_{B_{-} \min } .\end{cases}
$$

(2) Phase 2: Maximum Power Ascending for Higher Altitude. The phase of flight begins at time $t_{0}$ when the solar energy on the wings generated by solar cells at flight altitude $h_{1}$ is enough to sustain the climbing flight, and the stage finishes at time $t_{1}$ when the HSA reach the mission altitude $h_{2}$. During the whole phase, all the electrical energy generated by solar cells is applied for ascending. Thus, the proposed EMS of HSA satisfies

$$
\left\{\begin{array}{l}
\dot{E}=0, \\
P_{\mathrm{mppt}}=P_{\mathrm{prop}}=\frac{T \cdot v}{\eta_{\mathrm{ma}}} .
\end{array}\right.
$$

(3) Phase 3: Higher Altitude Charging Battery. This stage of flight starts at time $t_{1}$ when HSA get to the mission altitude $h_{2}$ for level flight, and ends at time $t_{2}$ when the solar energy charges the battery to the $Q_{B_{-} \max }$. In this stage, HSA only charge the battery at the mission altitude $h_{2}$, where some missions can be performed. The power supply mode is as follows: one part of the electricity generated by the solar cells is used to sustain the flight of the HSA, while the rest of the solar energy will charge the battery. At this stage, the proposed EMS of HSA is represented by the following formula:

$$
\dot{E}=\left\{\begin{array}{lr}
q_{\mathrm{max}}, & \left(P_{\mathrm{mppt}}-P_{\mathrm{prop}}\right) \eta_{b} \eta_{\mathrm{bm}} \geq q_{\mathrm{max}}, \\
\left(P_{\mathrm{mppt}}-P_{\mathrm{prop}}\right) \eta_{b} \eta_{\mathrm{bm}}, & 0<\left(P_{\mathrm{mppt}}-P_{\text {prop }}\right) \eta_{b} \eta_{\mathrm{bm}}<q_{\mathrm{max}}, \\
0, & Q_{B} \geq Q_{B_{-} \max } .
\end{array}\right.
$$

(4) Phase 4: Maximum Power Ascending for More Gravitational Potential Energy. This stage of flight begins at time $t_{2}$, with no task execution at mission altitude $h_{2}$, and finishes at time $t_{3}$ if the solar flux is zero. This stage consists of two processes: maximum power climbing when the $P_{\text {mppt }}$ of voltage converter is more than the $P_{\text {prop }}$, and powered gravity gliding when the $P_{\text {mppt }}$ could not satisfy the power consumption at its altitude. In this maximum power ascending phase, the HSA can make full use of the excess solar energy to gain the maximum altitude. The mode of power supply in this phase is as follows: all the solar energy is applied for powering the HSA for ascending and the battery is neither charged nor discharged. Therefore, the proposed EMS of HSA satisfies

$$
\left\{\begin{array}{l}
\dot{E}=0, \\
P_{\mathrm{mppt}}=P_{\text {prop }}=\frac{T \cdot v}{\eta_{\mathrm{ma}}}, \\
Q_{B}=Q_{B_{-} \max } .
\end{array}\right.
$$

(5) Phase 5: Longest Endurance Gliding. This phase of flight starts at time $t_{3}$ when there is no solar energy production by the solar cell and finishes at time $t_{4}$ when the HSA glides to the lower altitude $h_{1}$. During the entire phase, there is no solar energy to provide the HSA propulsion system and the thrust is zero. Besides, the battery will not supply any energy to the flight of HSA as the way to use gravity energy reserving. During the phase, the proposed EMS of HSA satisfies

$$
\left\{\begin{array}{l}
\dot{E}=0, \\
Q_{B}=Q_{B_{-} \max }, \\
T=0 .
\end{array}\right.
$$

To sum up, (12)-(16) constitute the EMS of HSA based on multiple flight phases.

2.4. Problem Implementation Frame and Solving Process. As shown in Figure 5 above, in phase 1, the HSA flight altitude is the altitude $h_{1}$, and the $P_{\text {mppt }}$ is basically close the zero, which is much smaller than $P_{\text {prop }}$ until $t_{0}$ time. During phase 2, the HSA climbs quickly between the altitude $h_{1}$ and altitude $h_{2}$, and the output power $P_{\text {mppt }}$ of the voltage converter is greater than the required power $P_{\text {prop. }}$. In phase 3 , the HSA only charges the battery at the mission altitude $h_{2}$ by the surplus from the $P_{\text {mppt }}$ to the $P_{\text {prop }}$. During phase 4, the output power $P_{\text {mppt }}$ is used to power the aircraft for 


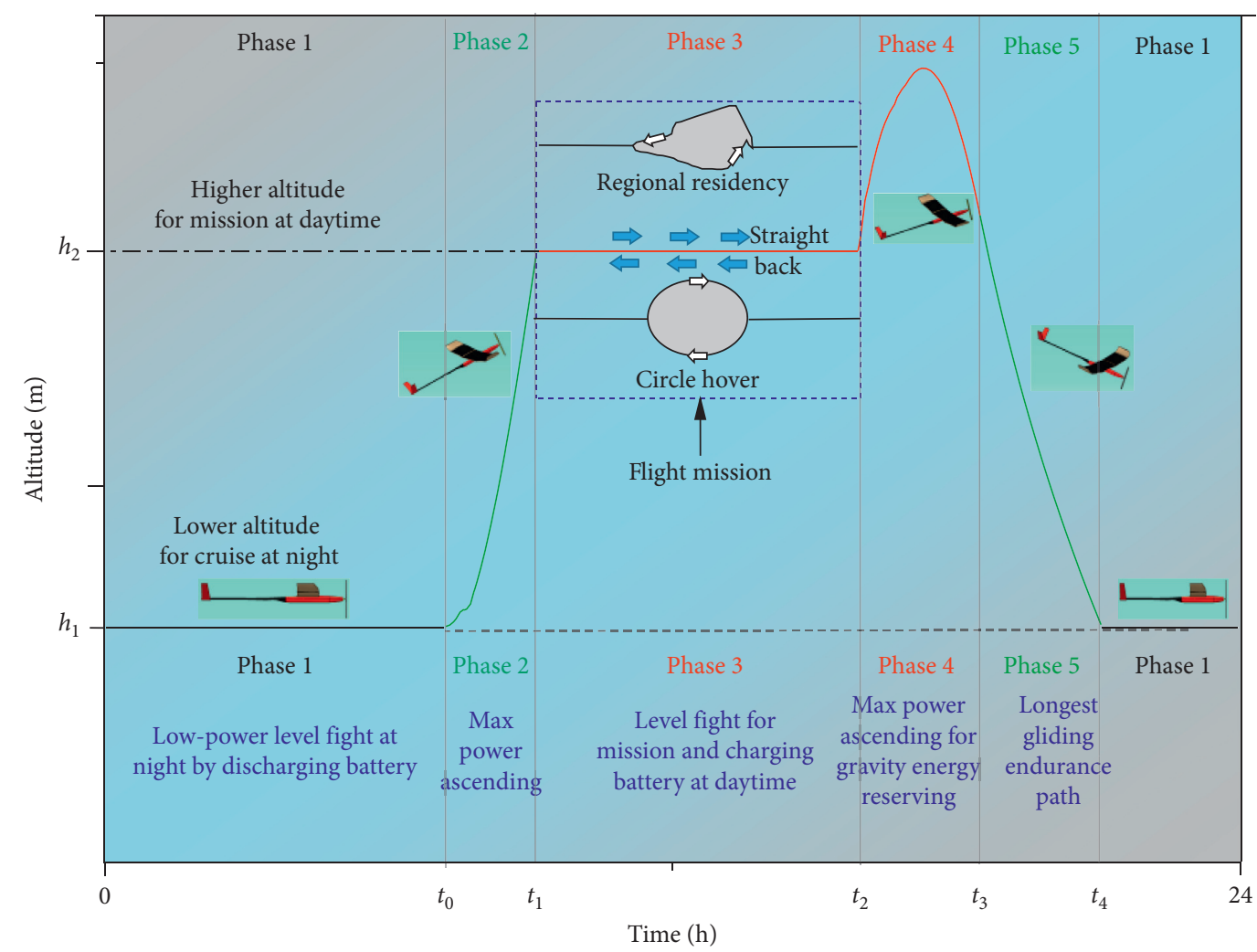

FIGURE 5: Schematic diagram of flight path of energy management strategy based on multiple flight phases.

ascending, which is equal to the propulsion system consumption $P_{\text {prop. }}$. At the last phase, the HSA glides to the cruising altitude $h_{1}$ under the condition that the $P_{\text {mppt }}$ and $P_{\text {prop }}$ are zero. Therefore, the three variables flight altitude $h$, propulsion power $P_{\text {prop }}$, and solar power $P_{\text {mppt }}$ can be used to determine which flight phase the aircraft is in. The efficiency of each component in energy management system must be also added in judging the phase. As a result, the HSA can adopt the appropriate proposed EMS at different phases. Table 2 shows the logical relationship to determine each flight phase according to $h, P_{\text {prop }}$, and $P_{\text {mppt }}$.

In phase 4 , the logical relationship about flight altitude $h$ is set as $h>h_{2}$, which may be seen to be unreasonable as the HSA may be at altitude $h_{1}<h<h_{2}$ before the sunset at weak irradiation. However, the study carefully conducts many flight path simulations based on our previous research [35], and it can be concluded that the flight altitude $h$ at $t_{3}$ time is greater than $h_{2}$, the same as the other research conclusions [5]. Meanwhile, the study will also take flight time $t>t_{\text {noon }}$ (the local standard time at noon) as the logical relationship in the proposed EMS model for following up further simulation. Therefore, the logical relationship in Table 2 can be reasonably used to build the new EMS model.

Based on the above introduction, a simulation application model of HSA about the proposed EMS calculation has been built as shown in Figure 6, where the aircraft kinematic and aerodynamic models are established based on (1) and (2). The solar irradiation model can be calculated according to (3)-(5).
In summary, the integrated model as shown in Figure 6 can calculate the new EMS in MATLAB/Simulink for HSA based on multiple flight phases, and it cloud be also used for the current EMS calculation with some modifications. The solving process is mainly the parameters initialization and calculation results analysis.

\section{Simulation and Discussion}

3.1. Simulation Parameters Setting. To illustrate the idea and advantage of the proposed EMS above, the results of numerical simulations on the current EMS and the new EMS have been calculated for comparison in this section, respectively. Firstly, the common atmospheric environment of the flight area has been analyzed for parameters settings, and the flight location of the HSA is set to Sanya, China $\left(109^{\circ} \mathrm{E}\right.$, $18.3^{\circ} \mathrm{N}$ ). Meanwhile, the initial configuration parameters of HSA are listed in Table 3; they are gained from Zephyr 7 because it has a detailed and comprehensive introduction in all the literature $[3,6,11,15]$ of HSA.

During the simulation process, the flight start time of Zephyr 7 is at $00: 00$ in June 20 . The conditions parameters are shown in Table 4.

3.2. Results and Discussion. As the initialization of the setting parameters above shows, the calculation results between the current EMS and the proposed EMS of the HSA have been obtained by the MATLAB/Simulink. The comparisons of altitude $h$, quantity of electricity in battery $Q_{B}$, battery charge 
TABLE 2: Logical relationship to determine each flight phase.

\begin{tabular}{lccccc}
\hline Variables & Phase 1 & Phase 2 & Phase 3 & Phase 4 & Phase 5 \\
\hline$h$ & $h=h_{1}$ & $h_{1}<h<h_{2}$ & $h=h_{2}$ & $h>h_{2}$ & $h>h_{1}$ \\
$P_{\text {mppt }} \& P_{\text {prop }}$ & $P_{\text {mppt }}<P_{\text {prop }}$ & $P_{\text {mppt }}=P_{\text {prop }}$ & $P_{\text {mppt }}>P_{\text {prop }}$ & $P_{\text {mppt }}=P_{\text {prop }} \quad P_{\text {mppt }}=P_{\text {prop }}=0$ \\
\hline
\end{tabular}

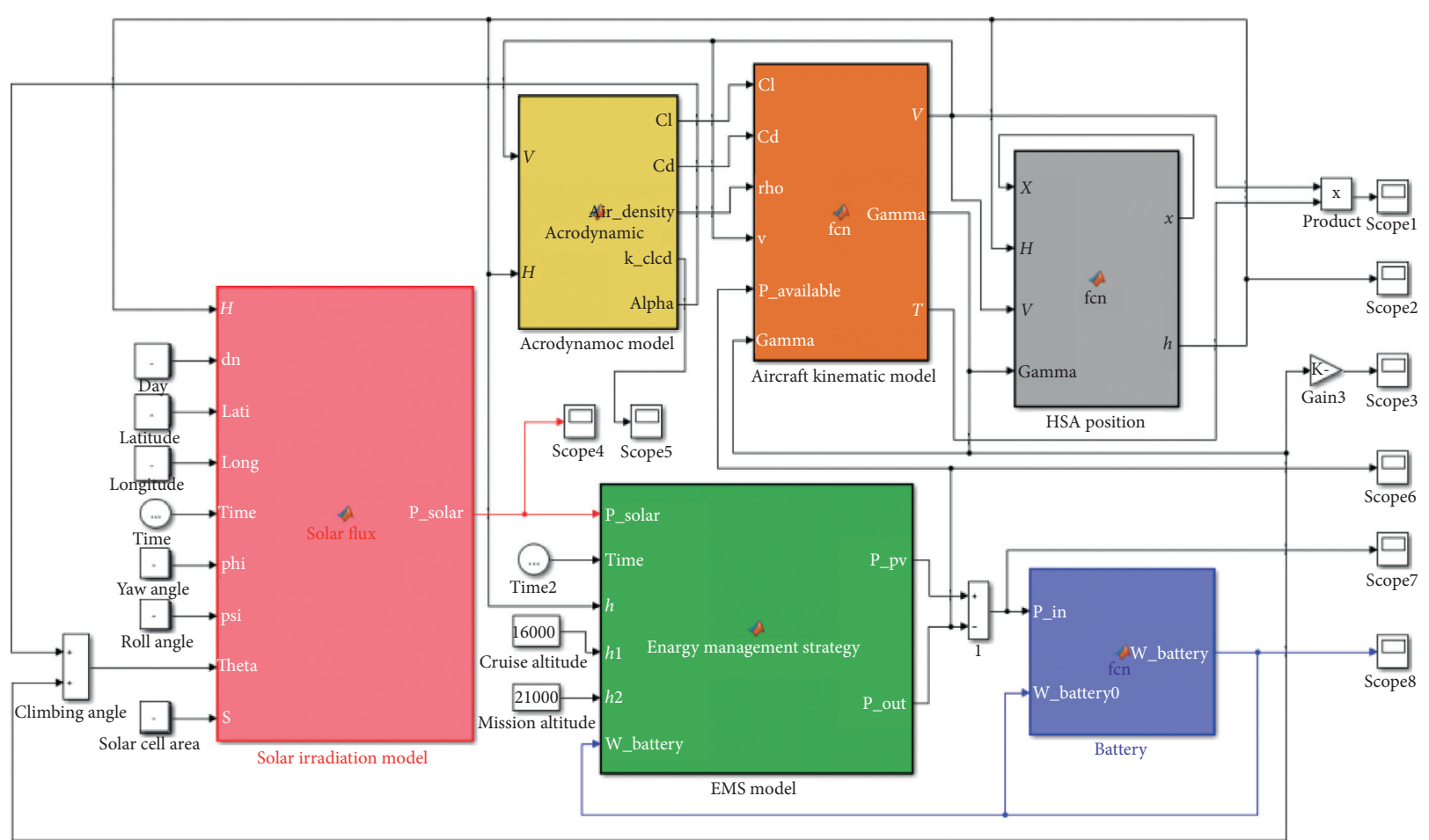

FigURE 6: Simulation model of proposed energy management strategy for solar-powered aircraft based on multiple flight stages.

TABLE 3: The initial configuration parameters for model.

\begin{tabular}{lccc}
\hline Parameters & Values & Units & Descriptions \\
\hline$m$ & 50 & $\mathrm{~kg}$ & Mass of aircraft \\
$Q_{B_{-} \text {max }}$ & 6 & $\mathrm{kWh}$ & Total power capacity of battery \\
$Q_{B_{-} 0: 00}$ & 3.5 & $\mathrm{kWh}$ & The initial electric quantity of battery \\
$b$ & 22.5 & $\mathrm{~m}$ & Span length \\
$S_{W}$ & 25.3 & $\mathrm{~m}^{2}$ & Wing area \\
$S_{\mathrm{SC}}$ & 16.2 & $\mathrm{~m}^{2}$ & Area of solar cells \\
\hline
\end{tabular}

TABLE 4: The simulation conditions parameters.

\begin{tabular}{lccc}
\hline Parameters & Values & Units & Descriptions \\
\hline$h_{1}$ & 16 & $\mathrm{~km}$ & Cruising altitude \\
$h_{2}$ & 21 & $\mathrm{~km}$ & Mission altitude \\
$d_{n}$ & 174 & - & day number \\
Lati & 18.3 & $\circ$ & Latitude of location \\
Long & 109 & $\circ$ & Longitude of location \\
\hline
\end{tabular}

and discharge power $E$, HSA speed $v$, pitching angel $\theta$, and propulsion power $P_{\text {prop }}$ are shown in Figures 7-11, respectively. Meanwhile, the red dotted line in each figure represents the available solar power $P_{\text {mppt }}$ in the wings of the HSA, which also starts at 00:00 in June 20. Generally, the green line represents the parameter in the proposed EMS based on multiple flight phases, and the black line means the parameter in the current EMS of level flight.

Figure 7 shows that the highest altitude of the HSA can reach about $30 \mathrm{~km}$ at time 14:46 based on the proposed EMS, which indicates that HSA makes full use of the solar energy to acquire higher altitude. As a result, the HSA can fly by gravitational potential energy at about 18:30-22:00 as shown in Figure 9.

Figure 8 and 9 show that the battery discharge time of the proposed EMS is 3.50 hours later than the current EMS. As a result, compared to the current EMS, about 22.9\% of energy surplus can be stored in battery with the proposed EMS during the long-endurance flight, which is conducive to the 


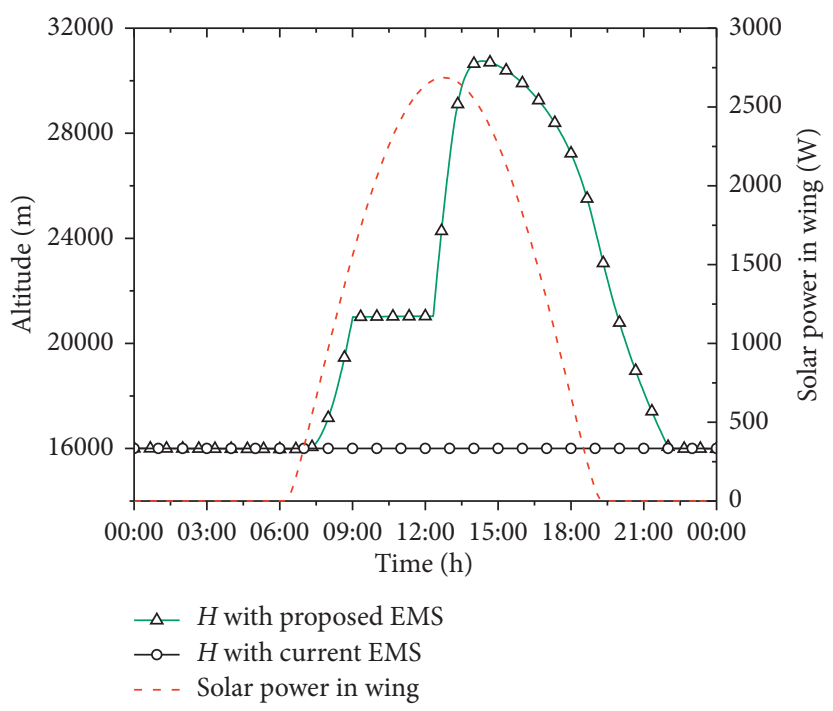

Figure 7: The comparison of altitude in the two energy management strategies.

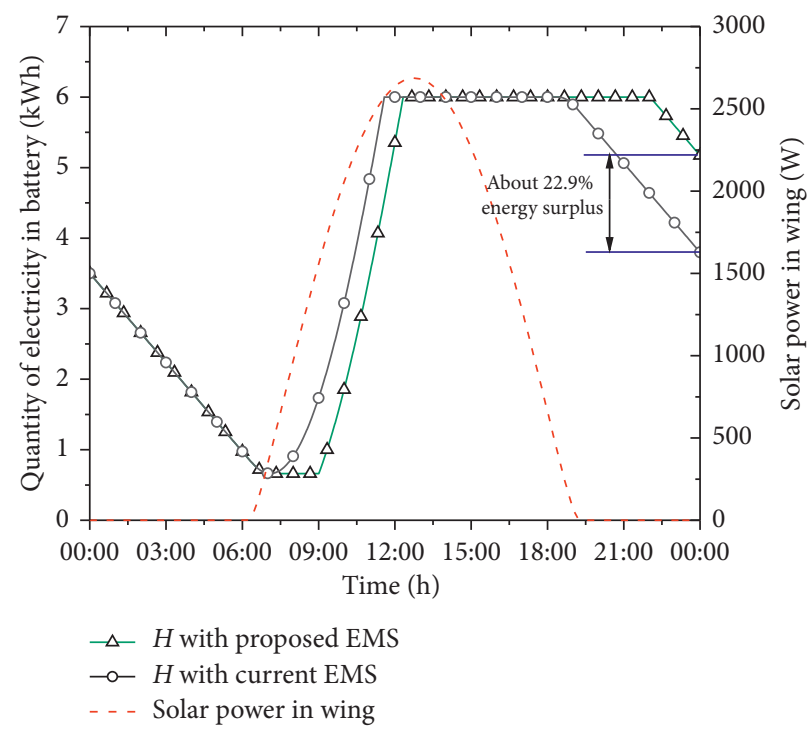

FIGURE 8: The comparison of quantity of electricity in battery in the two energy management strategies.

long-endurance flight of the HSA, especially the power consumption at night.

Figure 10 shows that the speed and pitching angle have the same trend as the flight altitude as shown in Figure 7, and the speed and pitching angle are constant in the current EMS and are changing in the proposed EMS. In addition, the changing of speed and propulsion power at sunrise and sunset in Figures 10 and 11 also indicate that the simulation results are reasonable with the proposed EMS based on multiple flight phases. Moreover, it is very attractive to analyze the changes like battery power and solar power at these special moments, which are helpful to understand the problem and validate the model.

In addition, a simulation example has been completed in different seasons (Spring Mar. 20, Summer Jun. 20, Autumn Sep. 20, and Winter Dec. 20) in Sanya. The simulation results are shown in Figures 12 and 13, and Figure 12 shows the variety of altitude and solar power in wings based on the proposed EMS in the four seasons. It can be concluded that the maximum flight altitude and the time of filling the battery will be strongly associated with the level of solar irradiation, especially in winter. Furthermore, Figure 13 displays the electric quantity of the batteries between the new EMS and the current EMS in the four seasons, and it clearly indicates that the current EMS cannot meet day and night flight for HSA in winter as the final electric quantity in battery is less than $Q_{B} 0: 00=3.5$ $\mathrm{kWh}$. What is more, the final electric quantity of the batteries with the proposed EMS in the four seasons is always larger than the $Q_{B_{-} 0: 00}$ with the current EMS. Therefore, the proposed EMS based on multiple flight phases is a very promising way to achieve the 


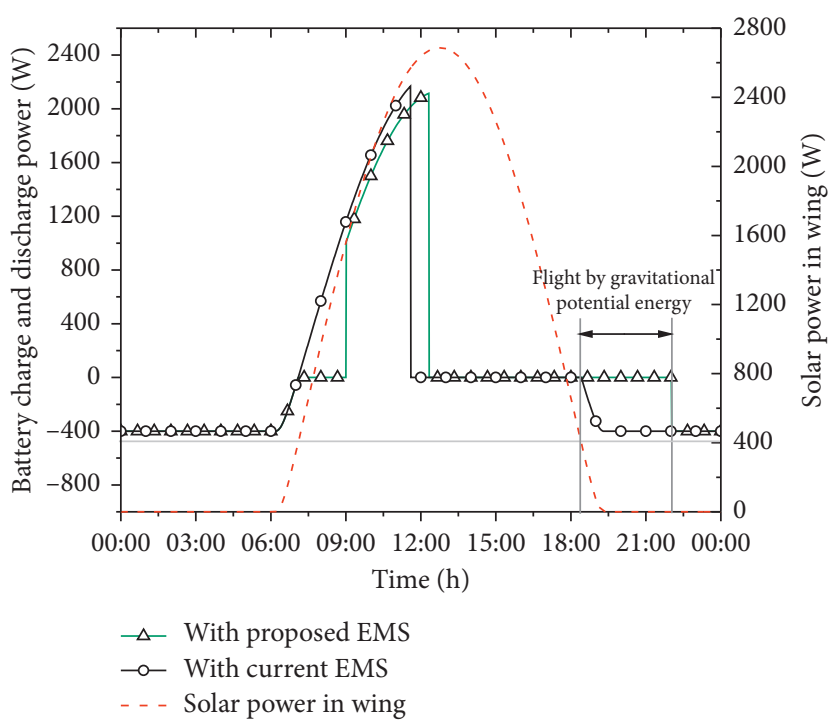

FIGURE 9: The comparison of battery charge and discharge power in the two energy management strategies.

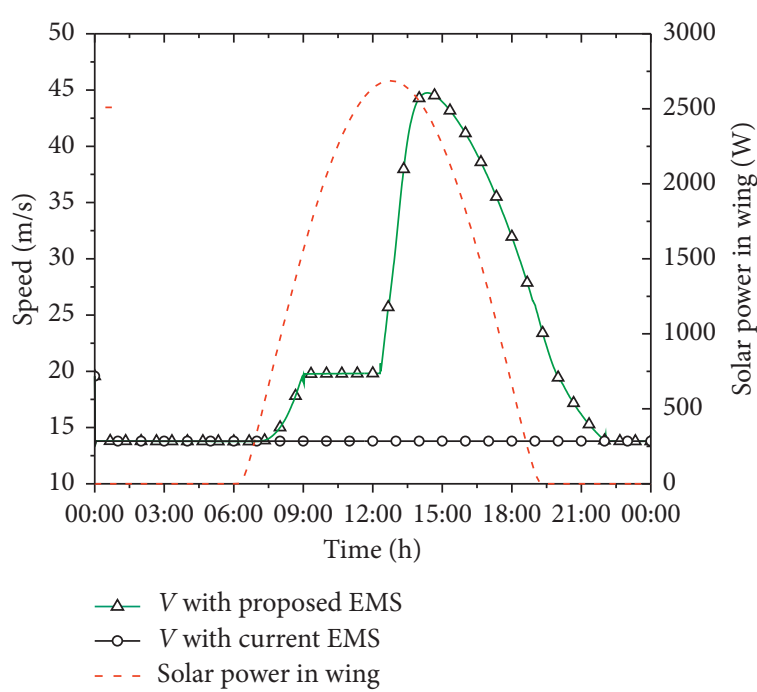

(a)

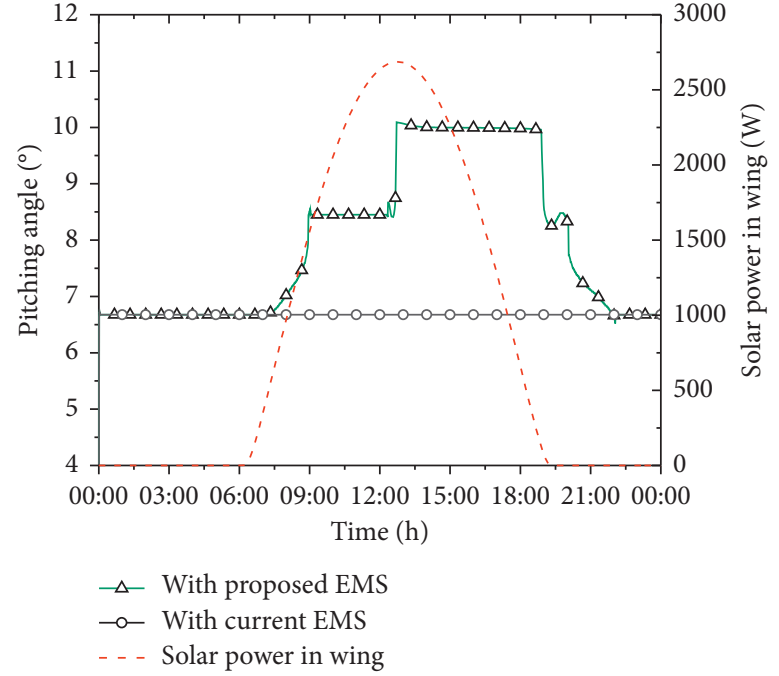

(b)

FIGURE 10: The comparison of speed and pitching angle in the two energy management strategies: (a) speed; (b) pitching angel.

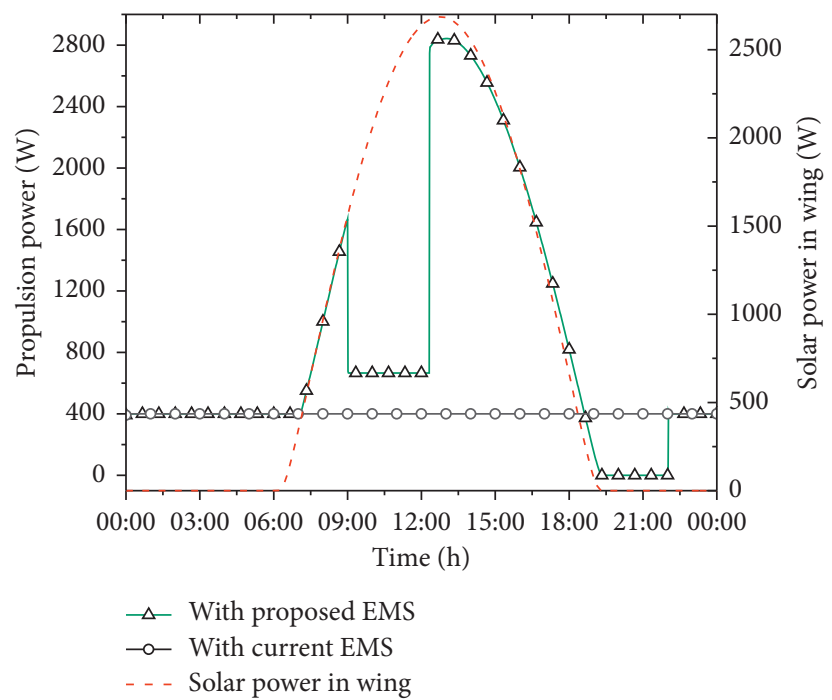

FIGURE 11: The comparison of propulsion power in the two energy management strategies. 


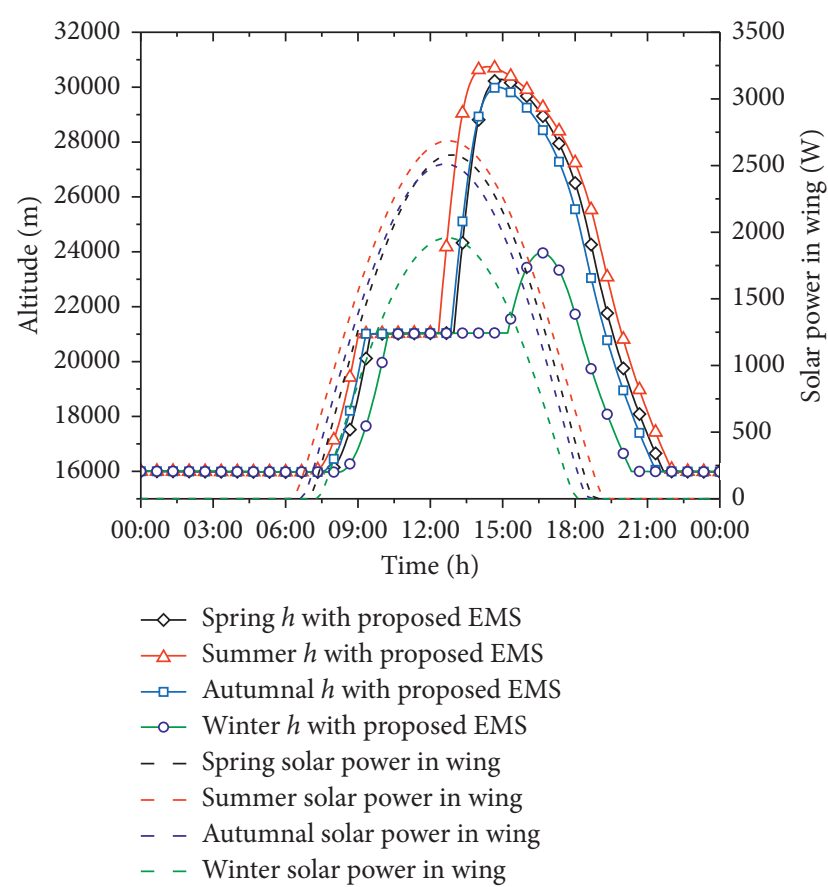

FIgURE 12: The variety of altitude and solar power in wing based on the proposed energy management strategy in the four seasons.

long-endurance goal on condition of lower solar irradiation and shorter daytime for HSA.

\section{Conclusions}

In this paper, a new EMS based on multiple flight phases including the mission altitude has been proposed to enhance the fight endurance for HSA. Compared to the current relatively simple EMS, the fight path of the proposed EMS has five phases, namely, the lower altitude level flight at night, the maximum power ascending for mission altitude, the level flight at mission altitude, the maximum power ascending for higher altitude, and the longest gliding endurance. Phase 2, phase 4 , and phase 5 could make good fitness and use of the gravity energy reserving strategy, and phase 3 shows and provides the possibility that HSA perform missions such as the regional residence, the straight back, and the circle hover just by more power consumption under the sufficient irradiation condition during the daytime. Besides, phase 3 can also give a smooth charging process relative to other research, which is more conducive to engineering applications.

The results of numerical simulations for Zephyr 7 on different season days have shown that the energy surplus with the new EMS is always more than the current EMS, since a fair amount of solar energy has been stored by the gravity energy reserving strategy based on this multiple flight phases. From the simulation results, the discharge time of Zephyr 7 battery can be delayed for about 3.50 hours with the proposed EMS on summer season days, and about $22.9 \%$ of energy surplus can be stored during the night, which is equal to reducing the battery weight from $16.0 \mathrm{~kg}$ to $12.3 \mathrm{~kg}$. In addition, the simulation results on winter season days

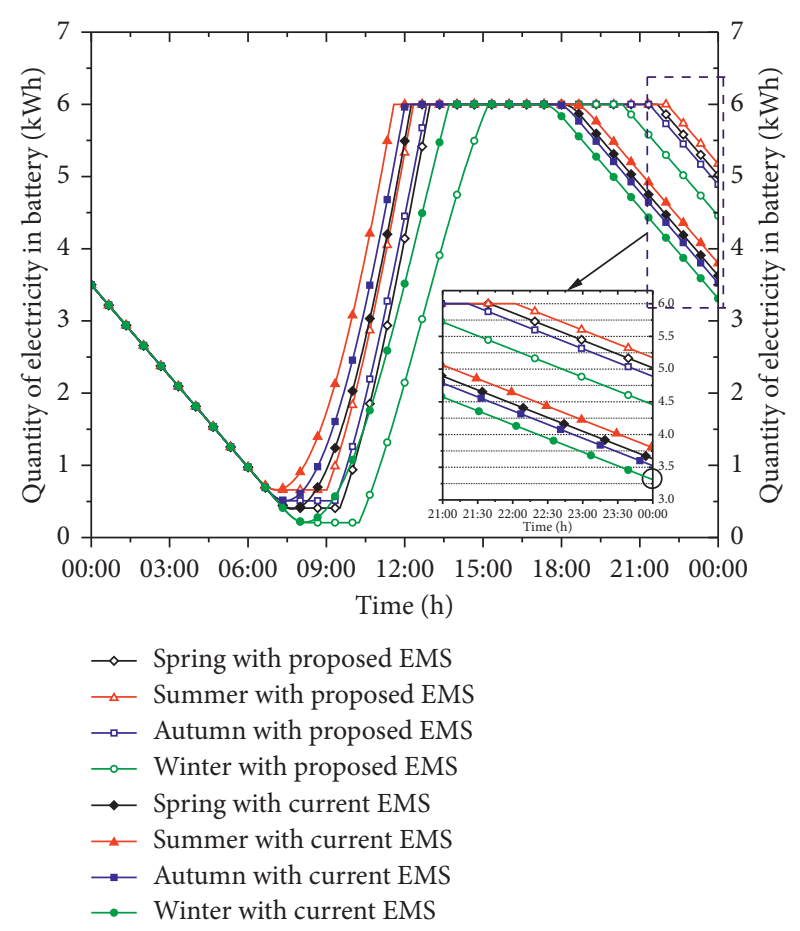

FIGURE 13: The comparison of electric quantity in battery in the four seasons based on the two energy management strategies.

clearly indicate that Zephyr 7 can fly across the day and night, but the current EMS cannot. Therefore, the new proposed EMS is a very promising way to achieve the longendurance goal for HSA when the flight conditions satisfy some constraints such as the deficiency of solar flux and the limit of battery mass.

It is also necessary to note that the study is a new twodimensional multiple-flight-phase EMS for HSA based on our previous research of $3 \mathrm{D}$ trajectory optimization, and the specific typical missions were not added to the simulation calculations because the purpose of this study is the new EMS and its calculations. Thus, some improved research will be studied and analyzed in the future.

\section{Data Availability}

The data used to support the findings of this study are included within the article.

\section{Conflicts of Interest}

The authors declare no conflicts of interest.

\section{Authors' Contributions}

M. S. and K. S. were responsible for conceptualization. M. S. contributed to methodology as well as writing, reviewing, and editing the manuscript. C. S. was responsible for software and data curation. M. S., Y. J., and C. S. participated in validation. K. S. helped with formal analysis, resources, visualization, and funding acquisition. Y. J. carried out investigation, supervision, and project administration in 
addition to writing the original draft of the manuscript. All authors have read and agreed to the published version of the manuscript.

\section{Acknowledgments}

This work was supported by the National Natural Science Foundation of China under Grant no. 51775021; the Key Laboratory of Aircraft Advanced Design Technology (Beihang University), Ministry of Industry; and the Fundamental Research Funds for the Central Universities under Grant no. YWF-18-BH-Y-165, the fifth youth top talent support program of Beihang University.

\section{References}

[1] O. R. Alvi, "Development of solar-powered aircraft for multipurpose application," in Proceedings of 2010 AIAA SDM Student Symposium, 51st AIAA/ASME/ASCE/AHS/ASC Structures, Orlando, FL, USA, April 2010.

[2] A. Noth, "Design of solar powered airplanes for continuous flight," Ph.D. thesis, Swiss Federal Institute of Technology Zurich, Zurich, Switzerland, 2008.

[3] X. Gao, "Research on high-altitude long-endurance flight based on energy storage by gravitational potential and energy extraction from wind shear," Ph.D. thesis, National University of Defense Technology, Changsha, China, 2014.

[4] "Zephyr pioneering the stratosphere," http://www.airbus. com.

[5] K. Sun, M. Zhu, L. Wang, and H. Liu, "Analysis of the hybird powered system for high-altitude unmanned aircraft," $A d$ vances in Materials Science and Engineering, vol. 2015, Article ID 380709, 9 pages, 2015.

[6] U. Papa, S. Ponte, and G. Core, "Conceptual design of a small hybird unmanned aircraft system," Journal of Advanced Transportation, vol. 2017, Article ID 9834247, 10 pages, 2017.

[7] T. Noll, J. Brown, M. Perez-Davis, S. Ishmael, G. Tiffany, and M. Gaier, Investigation of the Helios Protype Aircraft Mishap, Mishap Reprot, Hamptom, VA, USA, 2004.

[8] X.-Z. Gao, Z.-X. Hou, Z. Guo, R.-F. Fan, and X.-Q. Chen, "The equivalence of gravitational potential and rechargeable battery for high-altitude long-endurance solar-powered aircraft on energy storage," Energy Conversion and Management, vol. 76, pp. 986-995, 2013.

[9] Y. Huang, J. Chen, H. Wang, and G. Su, "A method of 3D path planning for solar-powered UAV with fixed target and solar tracking," Aerospace Science and Technology, vol. 92, pp. 831-838, 2019.

[10] Y. Zuo, P. Chen, L. Fu, Z. Gao, and G. Chen, "Advanced aerostructural optimization techniques for aircraft design," Mathematical Problems in Engineering, vol. 2015, Article ID 753042, 12 pages, 2015.

[11] X. Zhu, Generalized Energy Based Conceptual Design Method of Solar-Powered Airplane, Ph.D. thesis, National University of Defense Technology, Changsha, China, November 2014.

[12] R. Hannes, "Fly around the world with a solar powered airplane," in The 26th Congress of International Council of the Aeronautical Sciences, p. 8954p. 8954, Anchorage, Alaska, USA, 14-19 Semptember 2008.

[13] J. Gonzalo, D. López, D. Domínguez, A. García, and A. Escapa, "On the capabilities and limitations of high altitude pseudo-satellites," Progress in Aerospace Sciences, vol. 98, pp. 37-56, 2018.
[14] E. Cestino, "Design of solar high altitude long endurance aircraft for multi payload \& operations," Aerospace Science and Technology, vol. 10, no. 6, pp. 541-550, 2006.

[15] Z. Z. Annabel, A High Altitude Long Endurance Unmanned Air Vehicle, Master Thesis, University of Surrey, Guildford, UK, 2009.

[16] Nasa Dryden Flight Research Center, "Helios solar/fuel cell aircraft crashes," Fuel Cells Bulletin, vol. 8, p. 6, 2003.

[17] S. Herrería-Alonso, A. Suárez-González, M. Rodríguez-Pérez, R. F. Rodríguez-Rubio, and C. López-García, "A solar altitude angle model for efficient solar energy predictions," Sensors, vol. 20, no. 5, p. 1391, 2020.

[18] D. Scheiman, R. Holeisel, D. Edwards et al., "Enhanced endurance of a unmanned aerial vehicles using high efficiency $\mathrm{Si}$ and III-V solar cells," in Proceedings of the 2017 IEEE 44th Photovoltaic Specialist Conference, pp. 3514-3519, Washington, DC, USA, June 2017.

[19] V. Dwivedi, P. Kumar, A. K. Ghosh, and G. Kamath, "Selection of size of battery for solar powered aircraft," in Proceedings of the 11th IFAC Conference on Control Applications in Marine Systems, Robotics, and Vehicles, pp. 424-430, Opatija, Croatia, September 2018.

[20] X.-Z. Gao, Z.-X. Hou, Z. Guo, and X.-Q. Chen, "Reviews of methods to extract and store energy for solar-powered aircraft," Renewable and Sustainable Energy Reviews, vol. 44, pp. 96-108, 2015.

[21] J. Liao, Y. Jiang, J. Li et al., “An improved energy management strategy of hybrid photovoltaic/battery/fuel cell system for stratospheric airship," Acta Astronautica, vol. 152, pp. 727739, 2018.

[22] C. Shan, M. Lv, K. Sun, and J. Gao, "Analysis of energy system configuration and energy balance for stratospheric airship based on position energy storage strategy," Aerospace Science and Technology, vol. 101, Article ID 105844, 2020.

[23] J.-K. Shiau and D.-M. Ma, "Development of an experimental solar-powered unmanned aerial vehicle," Journal of the Chinese Institute of Engineers, vol. 38, no. 6, pp. 701-713, 2015.

[24] S. Schopferer, C. Liersch, M. Brizon, and S. Froese, "Evaluating the energy balance of high altitude platforms at early design stages," in Proceedings of the International Conference on Unmanned Aircraft Systems, pp. 170-177, Arlington, VA USA, June 2016.

[25] B. G. Gang, H. Kim, and S. Kwon, "Ground simulation of a hybrid power strategy using fuel cells and solar cells for highendurance unmanned aerial vehicles," Energy, vol. 141, pp. 1547-1554, 2017.

[26] S. C. Arum, D. Grace, P. D. Mitchell, M. D. Zakaria, and N. Morozs, "Energy management of solar-powered aircraftbased high altitude platform for wireless communications," Electronics, vol. 9, no. 1, p. 179, 2020.

[27] Y. Zhang, Y. Liu, and Y. Liu, "An hybrid dynamical modeling and control approach for energy saving of central air conditioning," Mathematical Problems in Engineering, vol. 5, pp. 1-12, Article ID 6389438, 2018.

[28] K. Wang, W. Wang, L. Wang, and L. Li, “An improved SOC control strategy for electric vehicle hybrid energy storage systems," Energies, vol. 13, no. 20, p. 5297, 2020.

[29] G. Pulido, G. Perales, J. Campaña, G. Gómez, and E. Velázquez, "Energy considerations for tracking in DC to DC power converters," Mathematical Problems in Engineering, vol. 2019, Article ID 1098243, 9 pages, 2019.

[30] K. Wang, X. Feng, J. Pang, J. Ren, C. Duan, and L. Li, "State of charge (SOC) estimation of lithium-ion battery based on adaptive square root unscented kalman filter," International 
Journal of Electrochemical Science, vol. 15, no. 9, pp. 94999516, 2020.

[31] J. Yan, H. Wang, S. Zhong, Y. Lan, and K. Huang, "Control strategy for the energy optimization of hybrid regenerative braking energy utilization system used in electric locomotive," Mathematical Problems in Engineering, vol. 2018, Article ID 2510487, 13 pages, 2018.

[32] X.-Z. Gao, Z.-X. Hou, Z. Guo, J.-X. Liu, and X.-Q. Chen, "Energy management strategy for solar-powered high-altitude long-endurance aircraft," Energy Conversion and Management, vol. 70, pp. 20-30, 2013.

[33] G. Sachs, J. Lenz, and F. Holzapfel, "Unlimited endurance performance of solar UAVs with minimal or zero electric energy storage," in Proceedings of the AIAA Guidance, Navigation, and Control Conference, Chicago, IL, USA, August 2009.

[34] J. Wu, H. Wang, Y. Huang, M. Zhang, and Z. Su, "Solarpowered aircraft endurance map," Journal of Guidance Control and Dynamics, vol. 42, pp. 687-694, 2018.

[35] M. Sun, X. Ji, K. Sun, and M. Zhu, "Flight strategy optimization for high-altitude solar-powered aircraft based on gravity energy reserving and mission altitude," Applied Sciences, vol. 10, no. 7, p. 2243, 2020. 